\title{
Constraining the Spin-Independent WIMP-Nucleon Coupling from Direct Dark Matter Detection Data
}

\section{Manuel Drees}

Physikalisches Institut der Universität Bonn, D-53115 Bonn, Germany

School of Physics, KIAS, Seoul 130-012, Republic of Korea

Bethe Center of Theoretical Physics, Universität Bonn, D-53115 Bonn, Germany

E-mail: dreesdth.physik.uni-bonn.de

\section{Chung-Lin Shan*}

School of Physics and Astronomy, Seoul National University, Seoul 151-747, Republic of Korea

E-mail: Cshan@hep1.snu.ac.kr

\begin{abstract}
Weakly Interacting Massive Particles (WIMPs) are one of the leading candidates for Dark Matter. For understanding the properties of WIMPs and identifying them among new particles produced at colliders (hopefully in the near future), determinations of their mass and their couplings on nucleons from direct Dark Matter detection experiments are essential. Based on our method for determining the WIMP mass model-independently from experimental data, we present a way to also estimate the spin-independent (SI) WIMP-nucleon coupling by using measured recoil energies directly. This method is independent of the as yet unknown velocity distribution of halo WIMPs. In spite of the uncertainty of the local WIMP density (of a factor of $\sim 2$ ), at least an upper limit on the SI WIMP-nucleon coupling could be given, once two (or more) experiments with different target nuclei obtain positive signals. In a background-free environment, for a WIMP mass of $100 \mathrm{GeV}$ its SI coupling on nucleons could in principle be estimated with a statistical error of only $\sim 15 \%$ with just 50 events from each experiment.
\end{abstract}

Identification of dark matter 2008

August 18-22, 2008

Stockholm, Sweden

* Speaker. 


\section{Introduction}

There is strong evidence that more than $80 \%$ of all matter in the Universe is dark (i.e., interacts at most very weakly with electromagnetic radiation and ordinary matter). The dominant component of this cosmological Dark Matter must be due to some yet to be discovered, non-baryonic particles. Weakly Interacting Massive Particles (WIMPs) $\chi$ with masses roughly between $10 \mathrm{GeV}$ and a few $\mathrm{TeV}$ are one of the leading candidates for Dark Matter (for reviews, see Refs. [1]).

Currently, the most promising method to detect many different WIMP candidates is the direct detection of the recoil energy deposited in a low-background laboratory detector by elastic scattering of ambient WIMPs on the target nuclei [2]. The differential rate for elastic WIMP-nucleus scattering is given by [1]:

$$
\frac{d R}{d Q}=\mathscr{A} F^{2}(Q) \int_{v_{\min }}^{v_{\max }}\left[\frac{f_{1}(v)}{v}\right] d v .
$$

Here $R$ is the direct detection event rate, i.e., the number of events per unit time and unit mass of detector material, $Q$ is the energy deposited in the detector, $F(Q)$ is the elastic nuclear form factor, $f_{1}(v)$ is the one-dimensional velocity distribution function of the WIMPs impinging on the detector, $v$ is the absolute value of the WIMP velocity in the laboratory frame. The constant coefficient $\mathscr{A}$ is defined as

$$
\mathscr{A} \equiv \frac{\rho_{0} \sigma_{0}}{2 m_{\chi} m_{\mathrm{r}, \mathrm{N}}^{2}},
$$

where $\rho_{0}$ is the WIMP density near the Earth and $\sigma_{0}$ is the total cross section ignoring the form factor suppression. The reduced mass $m_{\mathrm{r}, \mathrm{N}}$ is defined by

$$
m_{\mathrm{r}, \mathrm{N}} \equiv \frac{m_{\chi} m_{\mathrm{N}}}{m_{\chi}+m_{\mathrm{N}}}
$$

where $m_{\chi}$ is the WIMP mass and $m_{\mathrm{N}}$ that of the target nucleus. Finally, $v_{\min }=\alpha \sqrt{Q}$ is the minimal incoming velocity of incident WIMPs that can deposit the energy $Q$ in the detector with

$$
\alpha \equiv \sqrt{\frac{m_{\mathrm{N}}}{2 m_{\mathrm{r}, \mathrm{N}}^{2}}},
$$

and $v_{\max }$ is relared to the escape velocity from our Galaxy at the position of the Solar system.

\section{Estimating the SI WIMP-nucleon coupling}

Based on our work on the reconstruction of the (moments of the) velocity distribution of halo WIMPs [3], the integral over the one-dimensional WIMP velocity distribution on the right-hand side of Eq. (1.1), which is the minus-first moment of this distribution, can be estimated by [ [

$$
\left\langle v^{-1}\right\rangle\left(v\left(Q_{\min }\right), v\left(Q_{\max }\right)\right)=\int_{v\left(Q_{\min }\right)}^{v\left(Q_{\max }\right)}\left[\frac{f_{1}(v)}{v}\right] d v=\frac{1}{\alpha}\left[\frac{2 r\left(Q_{\min }\right) / F^{2}\left(Q_{\min }\right)}{2 Q_{\min }^{1 / 2} r\left(Q_{\min }\right) / F^{2}\left(Q_{\min }\right)+I_{0}}\right] .
$$

Here $v(Q)=\alpha \sqrt{Q}, Q_{(\min , \max )}$ are the minimal and maximal (cut-off) energies of the experimental data set, respectively, $r\left(Q_{\min }\right) \equiv(d R / d Q)_{Q=Q_{\min }}$, and $I_{n}\left(Q_{\min }, Q_{\max }\right)$ can be estimated by

$$
I_{n}\left(Q_{\min }, Q_{\max }\right)=\sum_{a} \frac{Q_{a}^{(n-1) / 2}}{F^{2}\left(Q_{a}\right)}
$$


where the sum runs over all events in the data set that satisfy $Q_{a} \in\left[Q_{\min }, Q_{\max }\right] .^{1}$ On the other hand, using the assumption that the spin-independent (SI) WIMP scattering cross section is the same for both protons and neutrons, the "pointlike" cross section $\sigma_{0}$ of Eq. (1.2) can be written as

$$
\sigma_{0}=\left(\frac{4}{\pi}\right) m_{\mathrm{r}, \mathrm{N}}^{2} A^{2}\left|f_{\mathrm{p}}\right|^{2}
$$

where $f_{\mathrm{p}}$ is the effective $\chi \chi \mathrm{pp}$ four-point coupling, and $A$ is the number of nucleons in the nucleus.

Substituting Eqs.(2.1) and (2.3) into Eq.1.1), it can easily be found that

$$
\left|f_{\mathrm{p}}\right|^{2}=\frac{1}{\rho_{0}}\left[\frac{\pi}{4 \sqrt{2}}\left(\frac{1}{\mathscr{E} A^{2} \sqrt{m_{\mathrm{N}}}}\right)\right]\left(m_{\chi}+m_{\mathrm{N}}\right)\left[\frac{2 Q_{\min }^{1 / 2} r\left(Q_{\min }\right)}{F^{2}\left(Q_{\min }\right)}+I_{0}\right] .
$$

Note that the factor $\mathscr{E}$ appearing in the denominator is the exposure of the experiment, which is dimensionless in natural units. It relates the actual counting rate to the normalized rate of Eq.(1.1). The WIMP mass $m_{\chi}$ on the right-hand side can be estimated by our method described in Ref. [ $₫$ ] using data from two experiments. $r\left(Q_{\min }\right)$ and $I_{0}$ can be estimated from one of the two data sets used for determining $m_{\chi}$ or from a third experiment. Recall that, due to the degeneracy between the local WIMP density $\rho_{0}$ and the WIMP-nucleus cross section $\sigma_{0}$, one can not estimate each one of them without using some assumptions. Hence, by using Eq.(2.4), one will need to accept an assumption of the local WIMP density $\rho_{0}{ }^{2}$

In Figs. 1, we show the reconstructed spin-independent WIMP-nucleon coupling $\left|f_{\mathrm{p}}\right|_{\text {rec }}^{2}$ as a function of the input WIMP mass $m_{\chi}$,in. Following our work on determination of the WIMP mass [凹], ${ }^{76} \mathrm{Ge}$ and ${ }^{28} \mathrm{Si}$ have been chosen as two targets for estimating $m_{\chi}$. In order to avoid calculating correlations between $m_{\chi}$ and $I_{0}$, a second independent data set with ${ }^{76} \mathrm{Ge}$ (left frame) or ${ }^{28} \mathrm{Si}$ (right frame) has been chosen for estimating $I_{0}$.

It can be seen in Figs. 1 that the reconstructed $\left|f_{\mathrm{p}}\right|_{\text {rec }}^{2}$ values are underestimated for WIMP masses $\gtrsim 100 \mathrm{GeV}$. For Ge this deviation is larger than for Si. This systematic deviation is caused by the underestimate of $I_{0}$. It is worse for heavier nuclei, where events with higher recoil energies can contribute more. This deviation of $I_{0}$ could be reduced by extending the maximal cut-off energy $Q_{\max }$ to higher energy range since the kinematic maximal cut-off energy is larger for heavier WIMP masses. On the other hand, although we used the same event number for both experiments the statistical error on $I_{0}$ estimated with $\mathrm{Si}$ is larger than that with Ge.

Nevertheless, it can be seen in Figs. 1 that, first, in spite of this systematic deviation, the true value of $\left|f_{\mathrm{p}}\right|_{\text {rec }}^{2}$ always lies within the $1 \sigma$ error interval. Second, for a WIMP mass of $100 \mathrm{GeV}$, one could in principle already estimate the SI WIMP-nucleon coupling with a statistical uncertainty of only $\sim 15 \%$ with just 50 events from each experiment.

\footnotetext{
${ }^{1}$ Note that the generalized moments $\left\langle v^{n}\right\rangle\left(v\left(Q_{\min }\right), v\left(Q_{\max }\right)\right)$ are independent of the local WIMP density, $\rho_{0}$, as well as of the WIMP-nucleus cross section, $\sigma_{0}$. Moreover, one does not need to know $f_{1}(v)$ in order to determine its moments via a generalization of Eq.(2.1). Every term needed in this paper e.g., $r\left(Q_{\min }\right)$ and $I_{n}\left(Q_{\min }, Q_{\max }\right)$, can be estimated either from a functional form of the scattering spectrum or from experimental data (i.e., the measured recoil energies) directly. More details about estimating $r\left(Q_{\min }\right), I_{n}\left(Q_{\min }, Q_{\max }\right)$, their statistical errors and the other formulae needed can be found in Refs. [3], 他.

${ }^{2}$ The most commonly used value for the local WIMP density is $\rho_{0} \approx 0.3 \mathrm{GeV} / \mathrm{cm}^{3}$. However, an uncertainty of a factor of $\sim 2$ has been usually adopted: $\rho_{0}=0.2-0.8 \mathrm{GeV} / \mathrm{cm}^{3}$.
} 


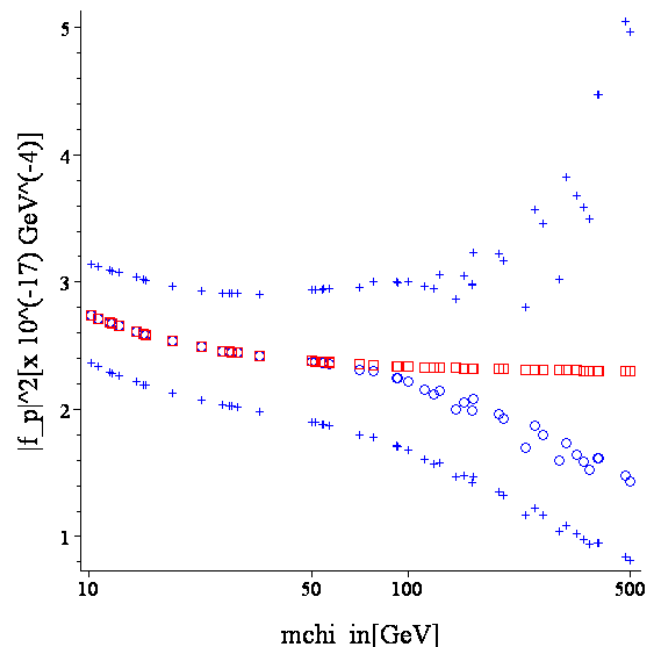

(a) ${ }^{76} \mathrm{Ge}$

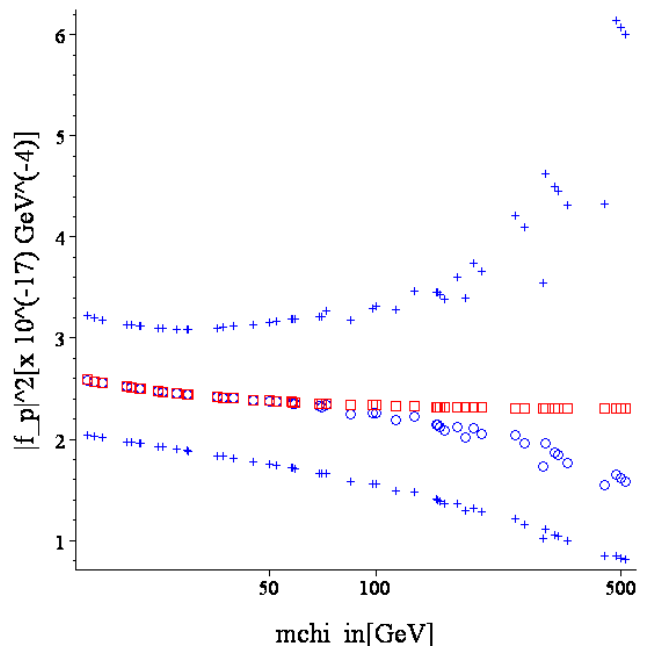

(b) ${ }^{28} \mathrm{Si}$

Figure 1: The reconstructed spin-independent WIMP-nucleon coupling $\left|f_{\mathrm{p}}\right|_{\mathrm{rec}}^{2}$ as a function of the input WIMP mass $m_{\chi, \text { in }}$. The open (red) squares indicate the input WIMP masses and the true values of the SI WIMP-nucleon couplings. The open (blue) circles and the (blue) crosses indicate the reconstructed SI WIMP-nucleon couplings and the $1 \sigma$ statistical errors. The theoretical predicted recoil spectrum for the shifted Maxwellian velocity distribution function [1], [3] with Woods-Saxon elastic form factor [5], [1] $\left(v_{0}=220 \mathrm{~km} / \mathrm{s}, v_{e}=231 \mathrm{~km} / \mathrm{s}\right)$ have been used. The WIMP-nucleon cross section has been set to be $10^{-8} \mathrm{pb} .{ }^{76} \mathrm{Ge}$ and ${ }^{28} \mathrm{Si}$ have been chosen as two targets for estimating $m_{\chi}$. A second (independent) data set with ${ }^{76} \mathrm{Ge}$ (left frame) or ${ }^{28} \mathrm{Si}$ (right frame) have been chosen as the third nucleus for estimating $I_{0}$. Each experimental data set has 50 events under the maximal cut-off energy $Q_{\max }$ chosen as $100 \mathrm{keV}$.

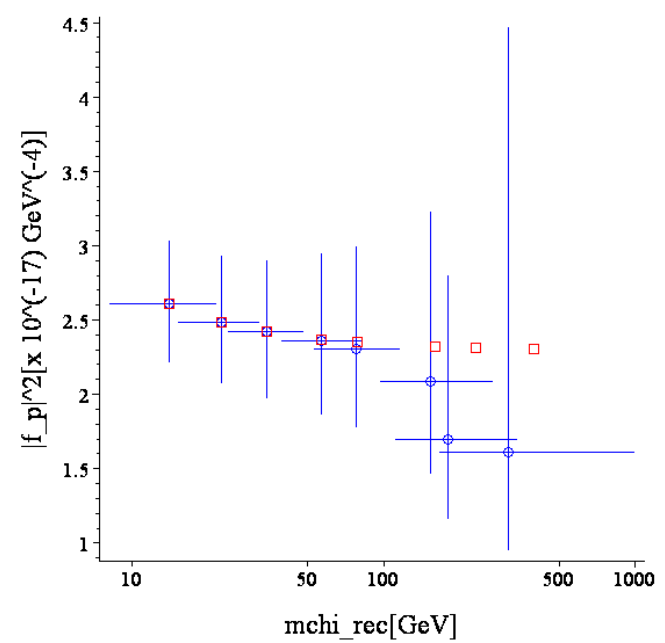

(a) ${ }^{76} \mathrm{Ge}$

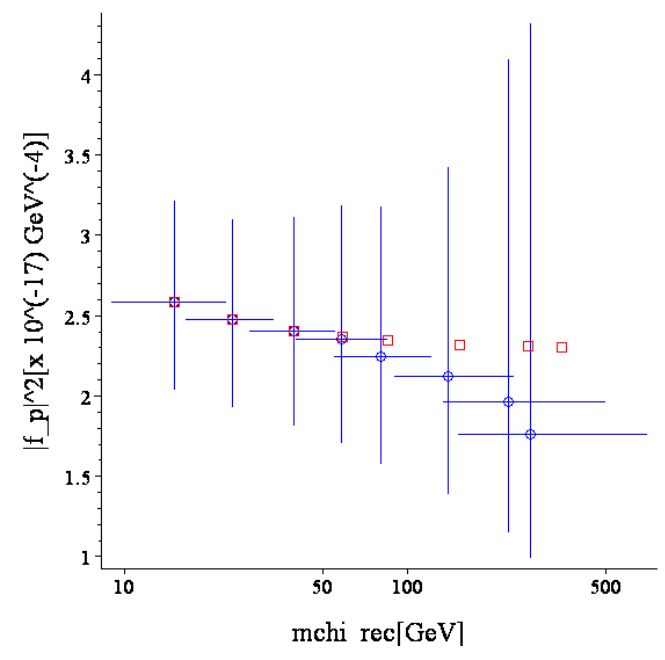

(b) ${ }^{28} \mathrm{Si}$

Figure 2: The reconstructed spin-independent WIMP-nucleon coupling $\left|f_{\mathrm{p}}\right|_{\text {rec }}^{2}$ and the reconstructed WIMP mass $m_{\chi \text {,rec }}$ on the cross section (coupling) v.s. WIMP mass plane. The open (red) squares indicate the input WIMP masses and the true values of the SI WIMP-nucleon couplings. The open (blue) circles indicate the reconstructed WIMP masses and the reconstructed SI couplings on nucleon. The horizontal and vertical solid (blue) lines show the $1 \sigma$ statistical errors on $m_{\chi \text {,rec }}$ and $\left|f_{\mathrm{p}}\right|_{\text {rec }}^{2}$, respectively. Parameters as in Fig. 1. 
Combining the estimate for $\left|f_{\mathrm{p}}\right|^{2}$ with that for $m_{\chi}$, in Figs. 2 we show the reconstructed SI coupling $\left|f_{\mathrm{p}}\right|_{\text {rec }}^{2}$ and the reconstructed WIMP mass $m_{\chi}$,rec on the cross section (coupling) v.s. WIMP mass plane. We emphasize that by our methods described in Ref. [ $[$ t and here, one can estimate $m_{\chi}$ and $\left|f_{\mathrm{p}}\right|^{2}$ separately without any assumption for the WIMP velocity distribution.

\section{Conclusions}

In this paper we have extended our method for determining the WIMP mass [ 4 ] to estimate the spin-independent WIMP-nucleon coupling from the elastic WIMP-nucleus scattering experiments. This method is independent of the velocity distribution of halo WIMPs as well as (practically) of the as yet unknown WIMP mass. The only information needed is the measured recoil energies from at least two experiments with different target nuclei and the local Dark Matter density as the unique assumption.

These information combined with the reconstructed WIMP mass will allow us not only to constrain the parameter space in different extensions of the Standard Model of particle physics, but also to identify WIMPs among new particles produced at colliders (hopefully in the near future). Once one is confident of this identification, one can use further collider measurements of the mass and couplings of WIMPs. Together with the reconstruction of the velocity distribution of halo WIMPs [3], this will then yield a new determination of the local WIMP density. On the other hand, knowledge of the WIMP couplings will also permit prediction of the WIMP annihilation cross section. Together with information on the WIMP density, this will allow to predict the event rate in the indirect Dark Matter detection as well as to test our understanding of the early Universe.

\section{Acknowledgments}

This work was partially supported by the Marie Curie Training Research Network "UniverseNet" under contract no. MRTN-CT-2006-035863, by the European Network of Theoretical Astroparticle Physics ENTApP ILIAS/N6 under contract no. RII3-CT-2004-506222, as well as by the BK21 Frontier Physics Research Division under project no. BA06A1102 of Korea Research Foundation.

\section{References}

[1] G. Jungman, M. Kamionkowski, and K. Griest, Phys. Rep. 267, 195 (1996); G. Bertone, D. Hooper, and J. Silk, Phys. Rep. 405, 279 (2005).

[2] P. F. Smith and J. D. Lewin, Phys. Rep. 187, 203 (1990); J. D. Lewin and P. F. Smith, Astropart. Phys. 6, 87 (1996).

[3] M. Drees and C. L. Shan, J. Cosmol. Astropart. Phys. 0706, 011 (2007).

[4] M. Drees and C. L. Shan, J. Cosmol. Astropart. Phys. 0806, 012 (2008).

[5] J. Engel, Phys. Lett. B 264, 114 (1991). 